\title{
TRANSMISIÓN DE TASAS DE INTERÉS BAJO EL ESQUEMA DE METAS DE INFLACIÓN: EVIDENCIA PARA COLOMBIA*
}

\author{
ÓsCAR REINALDo BECERRA \\ Fedesarrollo, Colombia \\ Luis Fernando Melo Velandia \\ Banco de la República, Colombia
}

This paper describes the dynamics of the monetary policy decisions enacted by the Central Bank of Colombia and the transmission to other interest rates, in order to identify the effectiveness and backwardness of such monetary policy measures. Based on a theoretical model, a VECX-MGARCH model is used to analyze the interactions among different interest rates and model the relationship between the volatilities of the endogenous variables of the model. Impulse-response functions suggest that the effect of a monetary shock on interest rates closely follow the objectives set by the Central Bank, both in sign and magnitude.

JEL: C32, E42, E44

Keywords: Transmisión de Tasas de Interés, Esquema de Metas de Inflación, Modelos VECX-MGARCH, Funciones de Impulso Respuesta en Volatilidad (VIRF)

\section{INTRODUCCIÓN}

Desde 2001 la política monetaria en Colombia se conduce bajo el esquema de metas de inflación (inflation targeting), según el cual el banco central busca "anclar" las expectativas de los agentes anunciando un rango de valores sobre el cual la tasa de inflación se debe encontrar al finalizar el año. En el caso colombiano, el Banco de la República (el banco central, BR de aquí en adelante) utiliza como instrumento de política sus tasas de interés de intervención, con el fin de modificar

\footnotetext{
* Los resultados y opiniones son responsabilidad exclusiva de los autores y su contenido no compromete al Banco de la República ni a su junta directiva. Los autores agradecen los comentarios y sugerencias de Luis Eduardo Arango, Rocío Betancourt, Munir Jalil y Hernando Vargas.

Email: Lmelovel@banrep.gov.co
} 
la tasa de interés interbancaria (su meta operativa) y a su vez influenciar las tasas de interés de plazos más largos, a partir de las cuales los individuos toman sus decisiones de consumo e inversión.

Este documento busca describir la dinámica de la transmisión de tasas de interés, entendiendo ésta como el mecanismo a través del cual las decisiones de política monetaria afectan las tasas de interés de los bancos comerciales. Como se discutirá más adelante, este tipo de aproximaciones tiene importantes implicaciones en la práctica ya que, por un lado, verifica la existencia de canales de transmisión comúnmente usados por los bancos centrales, como son los canales de demanda agregada y crédito y, además, cuantifica la efectividad (la magnitud de la respuesta de variables que se tienen como objetivos de política) y la velocidad del ajuste de los objetivos de política ante medidas de política monetaria.

El análisis de transmisión de tasas de interés es un tema que ha adquirido gran relevancia, en especial después de la implementación del esquema de metas de inflación, en el cual la tasa de interés de corto plazo se convierte en la meta operativa del banco central. Por ejemplo, Mojon (2000) verifica los efectos que tienen diferentes estructuras financieras sobre la transmisión de tasas de interés para varios países de la Unión Europea, mientras que Kok-Sorensen (2006) desarrolla un análisis similar para países de la Unión Europea, empleando nuevas técnicas econométricas de datos panel y cointegración. En el caso colombiano Betancourt et al. (2006) utilizan un modelo microfundamentado de la actividad bancaria que incluye una serie de variables de estado de la economía.

En líneas generales, este trabajo se divide en dos grandes secciones. En primer lugar, describe brevemente el esquema de metas de inflación usado en Colombia a la vez que plantea un modelo teórico en el cual se analizan las relaciones existentes entre las tasas de interés (activas y pasivas) de los bancos comerciales y la tasa de interés de política monetaria. De igual manera, el modelo identifica los mecanismos a través de los que las decisiones del banco central pueden afectar las decisiones de consumo y ahorro en la economía.

En segundo lugar, se estima un modelo multivariado de series de tiempo que describe la dinámica que existe entre las tasas de interés de créditos, de depósitos, interbancaria y la tasa de intervención del BR. Una de las principales diferencias de este documento radica en el uso de datos de alta frecuencia (semanales), los cuales permiten no sólo evaluar la magnitud y la velocidad con que las tasas de interés responden a los cambios de política monetaria, sino que también permiten analizar la reacción de la volatilidad de éstas ante choques de política. Este último punto se encuentra en línea con la idea que la efectividad (y credibilidad) de un banco central se incrementa cuando éste se desenvuelve en un ambiente más estable. En esta medida, si un banco central no influye en la volatilidad de las tasas de interés, fortalece la efectividad de sus medidas de política y contribuye a que los participantes en los mercados financieros identifiquen la postura de la política monetaria (Tuysuz, 2007).

La estructura del documento es como sigue. En la segunda sección se realizan algunas consideraciones sobre el funcionamiento de la política monetaria en Colombia bajo el esquema de metas de inflación; en la tercera sección se presenta un modelo teórico en el que se describe la actividad bancaria para un mercado de 
competencia perfecta, donde se establecen las relaciones entre las tasas de interés de los bancos comerciales y la tasa de política monetaria; la cuarta sección muestra los principales resultados de la estimación del modelo multivariado de tasas de interés. Finalmente, en la quinta sección se presentan las conclusiones.

\section{Política monetaria en Colombia}

Por mandato constitucional, la función básica del BR es velar por el mantenimiento del poder adquisitivo de la moneda. Para esto, desde comienzos de la década de los noventa, el BR implementó una serie de estrategias encaminadas a reducir la tasa de inflación, la cual, en promedio, osciló alrededor de $23 \%$ anual entre 1971 y 1990, alcanzando su valor máximo en este último año (32,4\%).

Durante el período 1992-1999, el eje fundamental de la política monetaria fue el uso de una meta intermedia monetaria (siguiendo el crecimiento de la base monetaria o de M1) junto con una banda cambiaria deslizante. Después de 1999, el BR decidió abandonar la banda cambiaria y comenzó la transición hacia un esquema de metas de inflación, en el que la tasa de interés de corto plazo se consolidó como el instrumento de política y el cumplimiento de una meta de inflación al finalizar el año se convirtió en el objetivo principal del banco central (Gómez et al., 2002).

En 2001 la política monetaria en Colombia terminó la transición hacia el esquema de metas de inflación. En la práctica, el BR busca "anclar" las expectativas de los agentes anunciando el valor sobre el cual la tasa de inflación se debe encontrar al finalizar cada año. Para cumplir con esta meta, el banco central utiliza como su principal instrumento las tasas de interés de las subastas de expansión (contracción) ${ }^{1}$, a través de las cuales suministra (contrae) liquidez al mercado a partir de la compra (venta) de títulos de deuda del Gobierno ${ }^{2}$.

Complementarias a las tasas de subastas, el Emisor mantiene dos tasas lombardas para suministrar (recibir) los faltantes (excesos) de liquidez de las entidades financieras después de las subastas (Huertas et al., 2005). Este sistema permitió establecer un rango sobre el cual oscila la tasa interbancaria (Gómez et al., 2002). Al igual que en el modelo planteado en la sección anterior, a través del control de las tasas de muy corto plazo, la autoridad monetaria busca afectar las tasas de interés de plazos más largos, modificando a su vez la demanda agregada y el nivel de precios.

El Gráfico 1 muestra la forma en que opera la estrategia de metas de inflación implementada en Colombia. La Junta Directiva del BR en su reunión mensual decide establecer las tasas de subasta y lombardas de expansión y contracción, con el fin de establecer el rango en el cual se debería mover la tasa de interés interbancario. Al variar la tasa interbancaria, esto afecta de diversas maneras la economía, entre

\footnotetext{
${ }^{1}$ La definición de estas series y las demás tasas de interés utilizadas en el documento se encuentra en el Anexo A.

${ }^{2}$ En el contexto del modelo presentado en la siguiente sección, el banco central altera la cantidad disponible de $B^{*}$ a través de la modificación de sus tasas de subasta de expansión y contracción.
} 
las cuales se destacan, cambios en las expectativas de inflación (un cambio en las expectativas futuras de inflación afecta la tasa de inflación observada), la tasa de cambio real y las tasas de interés de plazos más largos. Finalmente, los cambios en las tasa de interés de corto plazo afectan variables reales de la economía, inversión en el caso del canal de crédito y las exportaciones netas en el canal de tasa de cambio, afectando la producción y los precios (Mishkin, 1996).

\section{GRÁFICO 1 \\ POLÍTICA MONETARIA Y MECANISMOS DE TRANSMISIÓN EN COLOMBIA}

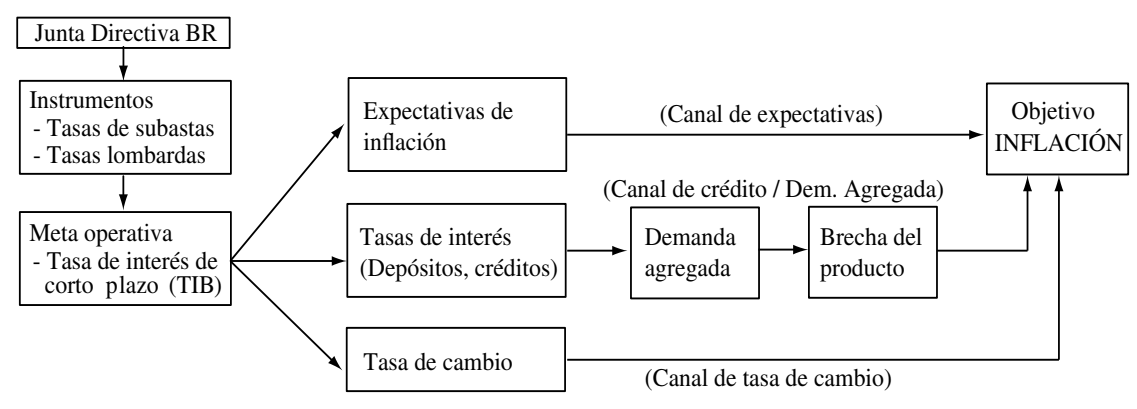

Ambiente institucional: Independencia, credibilidad y transparencia

Adaptado por Longworth (2000) y Huertas et al. (2005).

Como lo señalan Blejer y Leone (2000), un factor importante en este esquema es el ambiente institucional. Por un lado, para la implementación efectiva del esquema de metas de inflación, se requiere una completa independencia del banco central en el manejo de sus instrumentos, requisito que se satisface gracias a la independencia asignada al BR por la Constitución de 1991. En segundo lugar, la credibilidad desempeña un papel fundamental en este esquema, ya que mientras el banco central sea creíble, le será más fácil modificar las expectativas de los agentes en el mercado. Al respecto, la precisión de las metodologías usadas para pronosticar la inflación futura, aspecto clave para la toma de decisiones de política, resulta fundamental para cumplir las metas de inflación ${ }^{3}$. La transparencia y rendición de cuentas por parte de la autoridad monetaria permite generar un ambiente de confianza en las decisiones de política. Los informes trimestrales de inflación, la publicación de las minutas de las reuniones de la Junta Directiva desde mediados de 2007 y la simple verificación del cumplimiento de la meta

${ }^{3}$ Gómez et al. (2002) destacan que el desarrollo de las metodologías de pronóstico de inflación, junto con el modelo de mecanismos de transmisión, fueron determinantes para consolidar la transición hacia el esquema de metas de inflación. 
de inflación son algunos de los mecanismos que usa el BR como mecanismos de rendición de cuentas al país.

\subsection{Transmisión de tasas de interés y efectividad de la política monetaria}

Si bien los párrafos anteriores muestran el esquema a través del cual opera la política monetaria en Colombia, su efectividad sobre el control de la inflación dependerá en buena medida del grado de asociación que exista entre las tasas de interés controladas por el banco central y su meta operativa y, a su vez, de la respuesta que tengan las variables consideradas como claves para cada uno de los mecanismos de transmisión a las decisiones de política. En particular, la transmisión de tasas de interés (entendiendo ésta como la relación existente entre las tasas de intervención del BR, la tasa del mercado interbancario y las tasas de interés de los bancos comerciales) resulta fundamental para verificar la existencia de los canales de crédito y demanda agregada.

La transmisión de las tasas de interés ha sido un aspecto importante en los estudios de efectividad de la política monetaria ${ }^{4}$. Al respecto, la mayoría de los trabajos se concentran en cuantificar dos características de este tipo de transmisión. En primer lugar, se trata de identificar si el efecto de un incremento de la tasa de intervención sobre las demás tasas de interés es "completo" (uno a uno) o si, por el contrario, esta respuesta es inferior a la esperada por el banco central. Este aspecto resulta clave en la toma de decisiones por parte de la autoridad monetaria, ya que una correcta cuantificación de este efecto muestra la capacidad que tiene la política monetaria para influir sobre la economía a través de los canales de crédito y demanda agregada. En segundo lugar, la velocidad con que se ajustan las tasas de interés de mercado ante cambios en la tasa de intervención del banco central ofrece información adicional sobre la rapidez con que las autoridades monetarias pueden reaccionar a choques adversos en la economía.

Junto con la magnitud y la velocidad de la transmisión de las tasas de interés, un tercer aspecto relevante para determinar la efectividad de la política monetaria a través de este canal se encuentra en la volatilidad de la tasa de interés y el impacto que tienen las medidas del banco central sobre ésta. De hecho, una tarea importante para un banco central es la reducción de la volatilidad asociada a las medidas de política monetaria y esto se obtiene de forma más sencilla cuando un banco central es creíble (Tuysuz, 2007) ${ }^{5}$. Este es uno de los argumentos en que se sustenta la importancia de procurar la estabilidad de las tasas de interés, ya que una menor variabilidad de éstas reduce la incertidumbre a la que se enfrentan los agentes para la toma de decisiones de inversión y ahorro en el largo plazo, incrementa la

\footnotetext{
${ }^{4}$ Véanse, entre otros, los trabajos de Mojon (2000), Weth (2002), Bredin et al. (2002), Kok Sorensen y Werner (2006) y Betancourt et al. (2006).

5 El concepto de credibilidad y su relación con el manejo de política monetaria se encuentra en Blinder (1999).
} 
efectividad de los canales de transmisión de política monetaria ${ }^{6}$ y contribuye al aumento de la estabilidad macroeconómica (Amato y Laubach, 1999).

\section{El MODELO}

Como se mencionó, establecer cuáles son las relaciones que existen entre las tasas de interés al interior de una economía resulta de gran importancia, ya que esto permite verificar la existencia y efectividad de algunos de los canales de transmisión de la política monetaria. Para establecer dichas relaciones, este documento utiliza un modelo simple que describe la actividad bancaria en mercados de competencia perfecta, tal como se presenta en Freixas y Rochet (1997). Cabe anotar que si bien este documento utiliza una versión simple del modelo bajo competencia perfecta, las intuiciones derivadas de este modelo se mantienen bajo diferentes especificaciones ${ }^{7}$. A continuación, se presentan las principales características y resultados de este modelo.

\subsection{El problema de los bancos comerciales}

El modelo se fundamenta en un sistema bancario compuesto de $N$ bancos idénticos, cuya actividad económica consiste en brindar servicios financieros en los que los bancos obtienen depósitos de los hogares $\left(D_{n}, n=1, \ldots, N\right)$ y financian las necesidades de inversión de las firmas, a través de créditos $\left(L_{n}\right)$. Para cada uno de los bancos, los beneficios estarán determinados por

$$
\pi_{n}=r^{L} L_{n}+r^{M} B_{n}-r^{D} D_{n}-C\left(L_{n}, D_{n}\right)
$$

donde $r^{L}, r^{D}$ y $r^{M}$ son las tasas de interés de los préstamos, los depósitos y con la que los bancos obtienen recursos en el mercado interbancario, las cuales son tomadas como dadas por los bancos comerciales. La función $C\left(L_{n}, D_{n}\right)$ es la función de costos de administración, la cual se supone convexa, dos veces diferenciable e igual para todo $n$. Por último, $B_{n}$ representa la posición neta del $n$ - ésimo banco en el mercado interbancario, que se determina por la diferencia

$$
B_{n}=(1-d) D_{n}-L_{n}
$$

aquí, $d \in(0,1)$ es el coeficiente de reservas exigidas por el banco central, definido como una proporción fija de los depósitos. A partir de las ecuaciones (1) y (2), los

\footnotetext{
${ }^{6}$ Por ejemplo, Mojon (2000) encuentra evidencia que mayor volatilidad de la tasa de interés interbancaria reduce la efectividad de la transmisión de tasas de interés.

${ }^{7}$ Una discusión detallada de estos modelos se encuentra en Freixas y Rochet (1997, Cap. 3, pág. 51). Para el caso colombiano, Betancourt et al. (2006) utilizan un modelo microfundamentado de la actividad bancaria que incluye una serie de variables de estado de la economía con el fin de establecer relaciones más generales que las que se tratarán en este documento.
} 
bancos determinan la oferta de crédito y la demanda de depósitos que maximizan (1), dadas $r^{L}, r^{D}$ y $r^{M}$, las cuales se determinan en el mercado. Las condiciones de primer orden para maximizar (1) sujeto a (2) son

$$
\begin{aligned}
& \frac{\partial \pi_{n}}{\partial L_{n}}=r^{L}-r^{M}-\frac{\partial C}{\partial L_{n}}=0 \\
& \frac{\partial \pi_{n}}{\partial D_{n}}=(1-d) r^{M}-r^{D}-\frac{\partial C}{\partial D_{n}}=0
\end{aligned}
$$

de esta manera, los bancos buscarán los volúmenes de créditos y depósitos tal que los márgenes de intermediación $r^{L}-r^{M}$ y $(1-\mathrm{d}) r^{M}-r^{D}$ sean iguales a sus costos marginales de administración. Además, las ecuaciones presentadas en (3) indican que la oferta de créditos tendrá una relación positiva con $r^{L}$, mientras que incrementos en $r^{D}$ tendrán como resultado una disminución en la demanda de depósitos (Freixas y Rochet, 1997).

Reorganizando (3) se obtiene

$$
\begin{array}{r}
r^{L}-r^{M}=\frac{\partial C}{\partial L_{n}} \\
(1-d) r^{M}-r^{D}=\frac{\partial C}{\partial D_{n}}
\end{array}
$$

lo que establece una relación directa ente las tasas de interés del mercado interbancario y las tasas de los créditos y depósitos en la economía. En el óptimo, el costo marginal de un crédito debe ser igual a $r^{L}-r^{M}$. Más allá de esa diferencia, el banco comercial no otorgará más créditos. Un razonamiento similar aplica para la demanda de depósitos.

\subsection{Equilibrio competitivo}

Como se mencionó, los bancos proveen los servicios de intermediación financiera para transferir los recursos provenientes del ahorro de los hogares, $S$, hacia las necesidades de inversión de las firmas, $I$. En su versión más simple, el modelo supone que las empresas obtienen financiación únicamente a través del sistema bancario, por lo que la inversión será financiada en su totalidad por créditos y, en consecuencia, la inversión total coincidirá con la demanda de crédito. Con un razonamiento similar, en ausencia de bonos por parte de las empresas, el ahorro de los hogares se distribuirá entre los bonos emitidos por el gobierno, $B^{*}$, y los depósitos en los bancos comerciales ${ }^{8}$.

\footnotetext{
${ }^{8}$ Este modelo supone que estos dos tipos de activos son sustitutos perfectos para los hogares. En consecuencia, la tasa de interés pagada por los títulos del gobierno será igual a la tasa de depósitos, $r^{D}$.
} 
A partir de estos fundamentos, se define el equilibrio competitivo de la economía como el conjunto de tasas de interés $\left\{r^{L^{*}}, r^{D^{*}}, r^{M^{*}}\right\}$ y el conjunto de cantidades $\left\{D_{n}^{*}, L_{n}^{*}, I^{*}, S^{*}\right\}$, que satisfacen las siguientes condiciones:

Dado $\left\{r^{L^{*}}, r^{D^{*}}, r^{M^{*}}\right\}$, los bancos seleccionan la combinación $\left\{D_{n}^{*}, L_{n}^{*}\right\}$ que maximiza su función de beneficios, sujeto a una estructura de costos determinada y a la restricción de balance (2).

En el agregado, el conjunto de tasas de interés $\left\{r^{L^{*}}, r^{D^{*}}, r^{M^{*}}\right\}$ vacía los mercados de crédito (inversión), de depósitos (ahorro) e interbancario

$$
I\left(r^{L}\right)=\sum_{n=1}^{N} L_{n}\left(r^{L}, r^{D}, r^{M}\right)=L
$$

$$
S\left(r^{D}\right)=B^{*}+\sum_{n=1}^{N} D_{n}\left(r^{L}, r^{D}, r^{M}\right)=B^{*}+D
$$

$$
\begin{aligned}
\sum_{n=1}^{N} L_{n}\left(r^{L}, r^{D}, r^{M}\right) & =(1-d) \sum_{n=1}^{N} D_{n}\left(r^{L}, r^{D}, r^{M}\right) \\
L & =(1-d) D
\end{aligned}
$$

Esta última condición se obtiene debido a que la suma de las posiciones netas en el mercado interbancario es cero.

\subsection{Implicancias de política monetaria}

Si bien el modelo expuesto en los párrafos anteriores es relativamente simple, ofrece algunas herramientas para el análisis de medidas de política monetaria. En particular, éste destaca el uso de la tasa de interés interbancaria (ya sea directa o indirectamente) como instrumento de política. Nótese que cuando se supone que los costos marginales son constantes ${ }^{9}$, las relaciones representadas en (4) toman la forma

$$
\begin{aligned}
& r^{L}=r^{M}+c^{L} \\
& r^{D}=(1-d) r^{M}-c^{D}
\end{aligned}
$$

\footnotetext{
${ }^{9}$ Este supuesto es equivalente a que se presenten retornos constantes a escala (Freixas y Rochet, 1997).
} 
donde $c^{L}=\frac{\partial C}{\partial L_{n}}$ y $c^{D}=\frac{\partial C}{\partial D_{n}}$. En consecuencia, $L_{n}\left(r^{L}, r^{D}, r^{M}\right)=L_{n}\left(r^{L}, r^{M}\right)$ y $D_{n}\left(r^{L}, r^{D}, r^{M}\right)=D_{n}\left(r^{L}, r^{M}\right)$. Combinando (5) - (7) con (8) se obtiene

$$
S\left((1-d) r^{M}-c^{D}\right)-(1-d)^{-1} I\left(r^{M}+c^{L}\right)=B^{*}
$$

De esta manera, bajo el supuesto que los bonos del gobierno y los depósitos son sustitutos perfectos, si la autoridad monetaria varía la cantidad disponible de bonos en la economía, $B^{*}$, la tasa interbancaria (y, por ende, las tasas de créditos y depósitos) se ajustará de tal forma que se mantenga el equilibrio establecido en la condición (9). Así, la autoridad monetaria puede controlar la tasa interbancaria a través de operaciones de mercado abierto. Un aumento (disminución) en la cantidad de títulos del gobierno disponibles en la economía aumenta (disminuye) la tasa interbancaria de equilibrio. Por ejemplo, en el contexto presentado en la Sección 2, el banco central puede controlar la oferta de bonos a partir de una tasa de política $r^{S}$. En tal caso la ecuación (9) tomaría la forma

$$
S\left((1-d) r^{M}-c^{D}\right)-(1-d)^{-1} I\left(r^{M}+c^{L}\right)=B^{*}\left(r^{S}\right)
$$

la cual se supone que es exógena ${ }^{10}$ al problema y que, además, tiene una relación positiva con la cantidad de bonos disponibles en la economía. A partir de las consideraciones anteriores, se deriva el siguiente resultado:

Resultado 1. Bajo los supuestos presentados en esta sección, un incremento en la tasa de política implica un aumento de las demás tasas de interés de la economía (interbancaria, de créditos y depósitos). Adicionalmente, este incremento reducirá la cantidad de crédito y depósitos en la economía.

La prueba de este resultado es una simple extensión del resultado 3.2 de Freixas y Rochet (1997) para el caso en que la cantidad de bonos depende de una variable adicional y se deriva como sigue. Diferenciando totalmente la ecuación (10), se obtiene que

\footnotetext{
${ }^{10}$ Con relación a la exogeneidad de la variable de política monetaria, en Colombia, como se ilustra en las minutas de la Junta Directiva del Banco de la República (JDBR, la máxima autoridad monetaria), existe una serie de consideraciones adicionales a los niveles de tasa de interés en la toma de decisiones de política. En líneas generales, la JDBR fundamenta sus decisiones en i) la evolución actual de la inflación observada (total y sus principales categorías) y la esperada, capturadas por la encuesta de expectativas de inflación y tasa de cambio realizada mensualmente por el BR; ii) la evolución del panorama externo, el crecimiento de la economía y la demanda agregada, y iii) las proyecciones de inflación para los próximos meses. En consecuencia, este documento supone que el banco central determina de manera exógena su tasa de intervención al sistema. Desde mediados de 2007, las minutas de la JDBR se encuentran en el sitio web del BR (www.banrep.gov.co).
} 


$$
\frac{d r^{M}}{d r^{S}}=B^{* \prime}\left(r^{S}\right)\left[(1-d) S^{\prime}\left(r^{D}\right)-(1-d)^{-1} I^{\prime}\left(r^{L}\right)\right]^{-1}
$$

teniendo en cuenta que $B^{* \prime}\left(r^{S}\right)>0, S^{\prime}\left(r^{D}\right)>0, I^{\prime}\left(r^{L}\right)<0$, se concluye que $\frac{d r^{M}}{d r^{S}}>0$. El resultado sobre las demás tasas de interés es natural y se deriva de las condiciones presentadas en (8). Adicionalmente, derivando la condición de equilibrio (6) con respecto a la tasa de interés de política se tiene que

$$
\frac{d D}{d r^{S}}=B^{* \prime}\left(r^{S}\right)\left[\frac{(1-d)^{2} S^{\prime}\left(r^{D}\right)}{I^{\prime}\left(r^{L}\right)}-1\right]^{-1}
$$

al igual que en el caso anterior, $B^{* \prime}\left(r^{S}\right)>0, S^{\prime}\left(r^{D}\right)>0, I^{\prime}\left(r^{L}\right)<0$, en consecuencia $\frac{d D}{d r^{S}}<0$. Por último, a partir de la ecuación (7) se obtiene que

$$
\frac{d L}{d r^{S}}=(1-d) \frac{d D}{d r^{S}}<0
$$

Teniendo en cuenta los resultados anteriores, es posible verificar el efecto de un incremento de la tasa de interés de política sobre la economía. La condición (11) implica que un aumento de la tasa de intervención incrementa la tasa interbancaria, lo que a su vez determina un incremento en las tasas de interés de equilibrio de los depósitos y los créditos, una reducción de la inversión y una reasignación del ahorro hacia los títulos de deuda, disminuyendo así la cantidad el volumen de depósitos.

Bajo este esquema, el canal de transmisión de la política monetaria se deriva de la relación existente entre la tasa de política, la tasa de interés interbancaria y las tasas de interés de los créditos de los bancos, afectando de esta manera la inversión y la demanda agregada. Este canal es conocido como el canal de crédito bancario, el cual, en líneas generales, establece que cambios en la política monetaria afectan la cantidad de depósitos y créditos disponibles en la economía, alterando las decisiones de inversión, la demanda agregada y, finalmente, los precios (Mishkin, 1996).

\section{APROXIMACIÓN EMPÍRICA}

En las secciones anteriores se introdujeron algunas consideraciones que establecen las relaciones existentes entre las tasas de interés de depósitos y créditos y la tasa interbancaria, que está determinada en buena medida por los movimientos 
de las tasas de subastas y lombardas manejadas por el BR. Este documento se restringe a tratar de identificar las relaciones dinámicas entre las tasas de interés interbancaria, de créditos y de depósitos, ya que éstas se constituyen en la primera etapa del canal de transmisión de la política monetaria.

Siguiendo a Bernanke y Blinder (1992), las relaciones entre las tasas de interés se pueden aproximar por un modelo $V A R X^{11}$ en forma reducida, el cual es de la forma

$$
\mathbf{y}_{t}=\mathbf{A}_{0}+\mathbf{A}(Z) \mathbf{y}_{t-1}+\mathbf{M}(Z) \mathbf{p}_{t}+\mathbf{e}_{t}
$$

donde $\mathbf{y}_{t}=\left(r_{t}^{L}, r_{t}^{D}, r_{t}^{M}\right)^{\prime}$ es el vector que contiene los niveles de la tasa de colocación de los bancos comerciales (sin incluir las operaciones de tesorería), la tasa de depósitos a término fijo a 90 días y la tasa interbancaria, todas expresadas en términos nominales, reportadas por el BR. Por su parte, $\mathbf{p}_{t}=r_{t}^{S}$ representa la variable de política, en este caso la tasa de subasta de expansión del BR la cual se supone exógena al sistema. Todas las variables se presentan en frecuencia semanal, durante el período comprendido entre enero de 2001 y febrero de $2008^{12}$. Nótese que al incluir las variables en niveles se permite la existencia de relaciones de cointegración, las cuales, de acuerdo con lo establecido en las ecuaciones descritas en (8), deberían ser exactamente dos. Por último, $Z$ representa el operador de rezagos, es decir, $Z^{j} y_{t}=y_{t-j}$.

Con respecto a la especificación (14), vale la pena tener en cuenta ciertas consideraciones. Por un lado, la inclusión de la variable $\mathbf{p}_{t}$ permite modelar explícitamente el efecto de las decisiones de política monetaria sobre las demás tasas de interés. El polinomio $\mathbf{M}(Z)$ puede incluir tanto rezagos como adelantos, de manera que el modelo tiene en cuenta la posibilidad de que los individuos intenten "anticipar" los movimientos del banco central. Por último, el vector $\mathbf{e}_{t}$ representa el vector de innovaciones del sistema, el cual, teniendo en cuenta la posibilidad de que la volatilidad en el mercado no sea constante, se supone sigue una distribución con media cero y varianza condicional $H_{t}$.

Los resultados de las pruebas de especificación se presentan en el Anexo $\mathrm{B}^{13}$. De acuerdo con estas pruebas, se estimó un modelo $V E C X$ con dos relaciones de cointegración, incluyendo 10 rezagos de las variables endógenas y una relación contemporánea más 4 rezagos de la primera diferencia de la tasa subasta de expansión, sugeridos por los criterios de Schwarz (1978) y Hannan y Quinn (1979). Cabe destacar que cuando se incluyeron adelantos de la tasa de política en el modelo, éstos no resultaron significativos.

\footnotetext{
${ }^{11}$ Esta aproximación es comúnmente utilizada en la literatura. Véanse, por ejemplo, los trabajos de Dale y Haldane (1995) y Hülsewig et al. (2006), entre otros.

12 Una descripción detallada de la forma de cálculo de las series se encuentra en el Anexo A.

13 Teniendo en cuenta que en este caso el modelo VEC incluye variables exógenas, se hace necesario realizar una modificación sobre la estadística de la traza de Johansen (1988, 1991), sugerida por Boswijk y Doornik (2005). Adicional a las pruebas presentadas en el Anexo B, se realizaron pruebas de estabilidad sobre el modelo VECX implementadas por Lutkepohl (2004), de las que se concluye que el modelo es estable. Estos resultados se encuentran disponibles para los interesados vía correo electrónico.
} 
Una anotación importante se encuentra en que, de acuerdo con las pruebas de especificación del modelo, aunque los residuos del modelo no se encuentran autocorrelacionados, se encuentra evidencia de heterocedasticidad y no normalidad, resultado consistente con la modelación de datos de alta frecuencia (Bollerslev, 1986). En consecuencia, la estimación del modelo incluyó una segunda etapa en la que se estimó un modelo $G A R C H(1,1)$ multivariado $(M G A R C H)$ del tipo $B E K K$ sobre los residuales del modelo. Una descripción detallada de estos modelos se encuentra en el Anexo C.

\subsection{Efectos de un choque de política monetaria}

A partir del modelo $V E C X-M G A R C H$, se realizó un análisis de impulso respuesta del sistema ante un incremento permanente de 100 puntos básicos (p.b.) en la tasa subasta de expansión ${ }^{14}$, controlada por el BR. Teniendo en cuenta la no normalidad de los residuales del modelo, los intervalos de confianza fueron obtenidos a través de técnicas de bootstrapping, controlando por la heterocedasticidad estimada por el modelo $M G A R C H$. Los resultados se presentan en el Gráfico 2 donde se muestran las funciones de impulso y respuesta. Un incremento de 100 p.b. sobre la tasa subasta de expansión tiene un efecto positivo y significativo sobre la estructura de tasas de interés interbancaria, de depósitos y de créditos.

Por un lado, el efecto de la política monetaria sobre la tasa interbancaria es el esperado, tanto en signo como en magnitud. La tasa interbancaria es la tasa que responde de forma más rápida al choque en la tasa de subasta de expansión. En la semana que se presenta el choque, la respuesta contemporánea de la tasa interbancaria se encuentra alrededor de 80 p.b. para ubicarse alrededor de 100 p.b. después de la segunda semana. De esta manera, se confirma la idoneidad de la tasa interbancaria como meta intermedia de la política monetaria en Colombia, ya que debido a su estrecha relación con los instrumentos de política, al menos en una primera etapa, responde de manera rápida y estable ante choques de política.

Mientras tanto, si bien el efecto después de un año de un choque de política monetaria sobre las tasas de interés de depósitos y de créditos es similar (alrededor de 100 p.b.), su dinámica resulta diferente. En efecto, la tasa de certificados de depósito a 90 días reacciona de manera suave ante el choque de política durante todo el período. Los incrementos más significativos se encuentran alrededor del primer trimestre y posteriormente su ritmo de aumento tiende a moderarse.

Por su parte, la tasa de créditos muestra un comportamiento más errático las primeras semanas después del choque, para posteriormente estabilizarse después de un trimestre. Una posible explicación de este resultado se encuentra asociada a la duración media de los depósitos y créditos. En efecto, de acuerdo con las series incluidas en el documento, el plazo promedio de los créditos otorgados por los bancos es superior a un año, mientras que el plazo de los depósitos considerados es de 90 días. En consecuencia, los pasivos de los bancos (depósitos) pueden ajustarse de manera más rápida que los activos (créditos).

${ }^{14}$ Véase Lutkepohl (2005). 


\section{GRÁFICO 2 \\ FUNCIONES DE IMPULSO RESPUESTA}

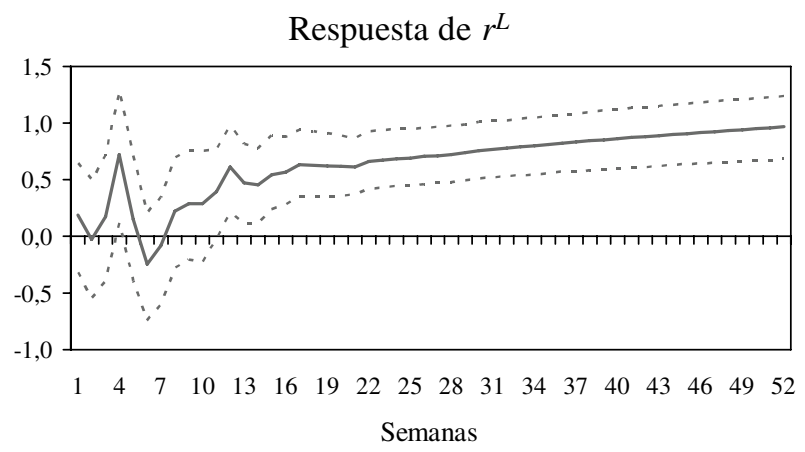

Respuesta de $r^{D}$

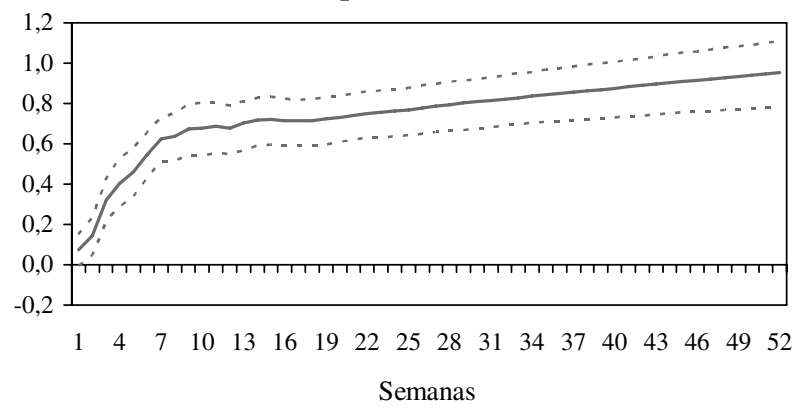

Respuesta de $r^{M}$

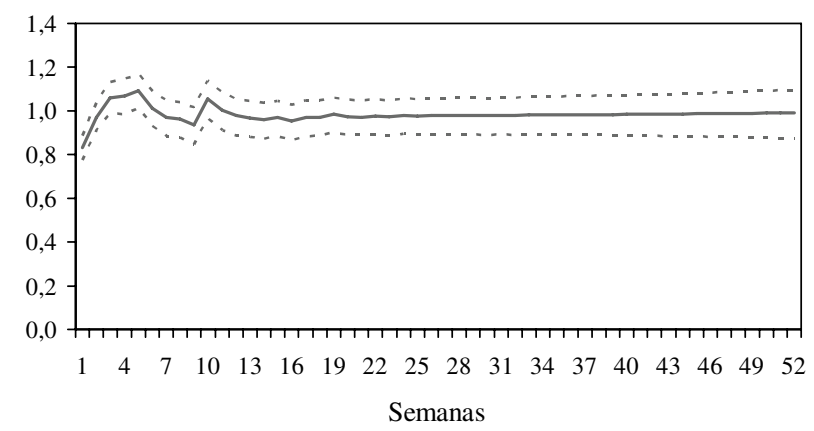

Nota: Función que muestra la respuesta de las series ante un choque permanente de 100 p.b. en la tasa subasta de expansión (RS). Intervalos de confianza de $90 \%$ obtenidos a través de técnicas bootstrapping con 1.000 replicaciones. 
Por último, un resultado importante que predice el modelo de la Sección 2 que no se ratifica completamente en el análisis de impulso respuesta se encuentra en que el efecto de largo plazo de un choque de política monetaria sobre la tasa de interés de los créditos es superior al impacto de este mismo choque sobre la tasa de depósitos. De esta manera, si bien en el corto plazo la dinámica de las tasas de créditos y depósitos difiere, en el largo plazo tiende a estabilizarse y ser de la misma magnitud.

Con respecto a estos resultados, cabe destacar que la magnitud de las funciones de impulso respuesta se encuentran en línea con los resultados obtenidos en otros trabajos realizados para el caso colombiano. Por ejemplo, Huertas et al. (2005) utilizan ejercicios de estadística descriptiva para calcular el impacto de la tasa de subasta de expansión sobre la tasa interbancaria y la tasa de depósitos y, si bien los resultados con respecto a la tasa de depósitos difieren levemente, sus conclusiones son similares a las que se derivan del Gráfico 2.

4.2 Efectos de un choque de política monetaria sobre la volatilidad de las tasas de interés

Al igual que en el caso anterior, también es posible establecer cuál es el impacto de un choque de política monetaria sobre la volatilidad de las tasas de interés. Como se mencionó, incrementos en la volatilidad de las tasas de interés asociados a las decisiones de política monetaria dificultan la acción de los bancos centrales, ya que ante tasas de interés más volátiles, las decisiones de consumo e inversión de los agentes deben ser tomadas bajo escenarios de mayor incertidumbre.

Con este fin, se utilizó la aproximación empírica de Hafner y Herwartz (2006), en la que se calculan las funciones de impulso respuesta sobre la varianza condicional (VIRF por sus siglas en inglés) de la serie a partir de los parámetros del modelo MGARCH estimado. Una discusión detallada de la implementación de las VIRF se encuentra en el Anexo D. Cabe anotar que, para realizar una interpretación más apropiada, Hafner y Herwartz (2006) proponen escalar cada elemento de la VIRF con respecto a las volatilidades condicionales estimadas en el período donde se realiza el choque. En consecuencia, las funciones VIRF dependerán de este período y se interpretan como desviaciones porcentuales de la volatilidad condicional de las series en el período del choque.

A partir de estos resultados es posible analizar la reacción, en términos de volatilidad, del mercado de depósitos y créditos ante cambios en la política monetaria, los que en este contexto se representan a partir de un choque en el término de innovación asociado al proceso generador de datos de la tasa de interés interbancaria, teniendo en cuenta la estrecha relación existente entre esta tasa y la tasa de intervención del banco central, descrita en la Sección 2 y ratificada por los resultados presentados en la Sección 4.1.

Las funciones VIRF estimadas se exhiben en el Gráfico 3. Para este caso, se presenta la respuesta de las volatilidades de las series ante un choque en el término de perturbación asociado a la tasa interbancaria de 25 puntos básicos, para la primera semana de agosto de 2002. En general, la respuesta ante un choque de 


\section{GRÁFICO 3 \\ FUNCIONES DE IMPULSO RESPUESTA EN VARIANZA}

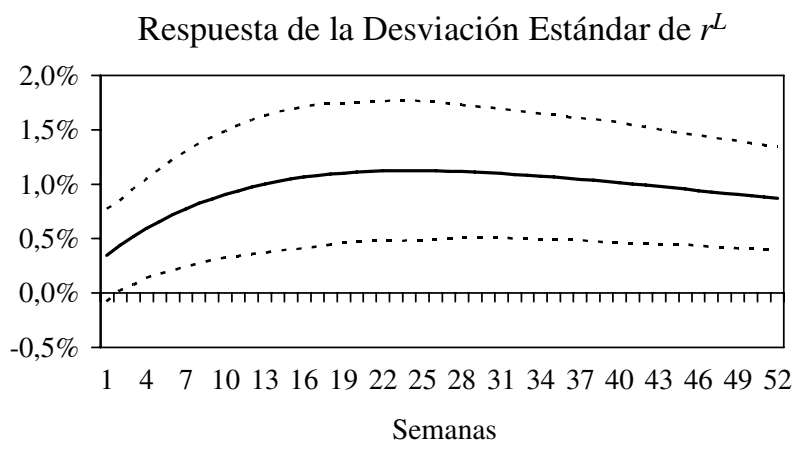

Respuesta de la Desviación Estándar de $r^{D}$
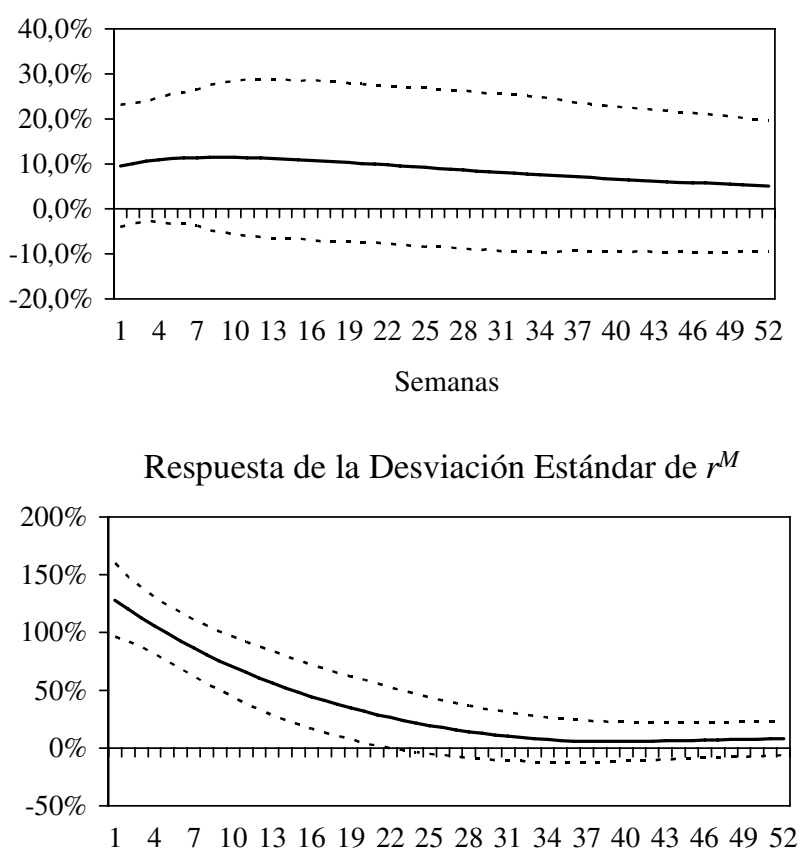

Semanas

Nota: Función VIRF que muestra la respuesta en la desviación estándar de las series ante un choque de 25 p.b. en la tasa interbancaria (RM) en la primera semana de agosto de 2002. Intervalos de confianza de $90 \%$ obtenidos a través de técnicas bootstrapping con 1.000 replicaciones. 


\section{CUADRO 1 \\ FUNCIONES VIRF PARA CHOQUES EN DIFERENTES PERÍODOS \\ DE LA MUESTRA \\ (Intervalos de confianza entre paréntesis)}

\begin{tabular}{|c|c|c|c|c|c|c|}
\hline \multirow{2}{*}{$\begin{array}{l}\text { Choque } \\
\text { (dd-mm-aa) }\end{array}$} & \multicolumn{6}{|c|}{ VIRF para la variable RL } \\
\hline & Semana 1 & Semana 4 & Semana 13 & Semana 26 & Semana 39 & Semana 52 \\
\hline 09-08-2002 & $\begin{array}{c}0,3 \% \\
(-0,1 \%, 0,8 \%)\end{array}$ & $\begin{array}{c}0,6 \% \\
(0,1 \%, 1 \%)\end{array}$ & $\begin{array}{c}1 \% \\
(0,4 \%, 1,6 \%)\end{array}$ & $\begin{array}{c}1,1 \% \\
(0,5 \%, 1,8 \%)\end{array}$ & $\begin{array}{c}1 \% \\
(0,5 \%, 1,6 \%)\end{array}$ & $\begin{array}{c}0,9 \% \\
(0,4 \%, 1,3 \%)\end{array}$ \\
\hline $26-12-2003$ & $\begin{array}{c}0,4 \% \\
(-0,1 \%, 0,8 \%)\end{array}$ & $\begin{array}{c}0,6 \% \\
(0,2 \%, 1,1 \%)\end{array}$ & $\begin{array}{c}1,1 \% \\
(0,4 \%, 1,8 \%)\end{array}$ & $\begin{array}{c}1,2 \% \\
(0,5 \%, 1,9 \%)\end{array}$ & $\begin{array}{c}1,1 \% \\
(0,5 \%, 1,7 \%)\end{array}$ & $\begin{array}{c}1 \% \\
(0,4 \%, 1,5 \%)\end{array}$ \\
\hline 06-05-2005 & $\begin{array}{c}0,4 \% \\
(-0,1 \%, 0,9 \%)\end{array}$ & $\begin{array}{c}0,7 \% \\
(0,2 \%, 1,2 \%)\end{array}$ & $\begin{array}{c}1,2 \% \\
(0,4 \%, 1,9 \%)\end{array}$ & $\begin{array}{c}1,3 \% \\
(0,6 \%, 2 \%)\end{array}$ & $\begin{array}{c}1,2 \% \\
(0,5 \%, 1,8 \%)\end{array}$ & $\begin{array}{c}1 \% \\
(0,4 \%, 1,6 \%)\end{array}$ \\
\hline 22-09-2006 & $\begin{array}{c}0,4 \% \\
(-0,1 \%, 0,9 \%)\end{array}$ & $\begin{array}{c}0,7 \% \\
(0,2 \%, 1,2 \%)\end{array}$ & $\begin{array}{c}1,2 \% \\
(0,5 \%, 1,9 \%)\end{array}$ & $\begin{array}{c}1,3 \% \\
(0,6 \%, 2,1 \%)\end{array}$ & $\begin{array}{c}1,2 \% \\
(0,6 \%, 1,9 \%)\end{array}$ & $\begin{array}{c}1 \% \\
(0,5 \%, 1,6 \%)\end{array}$ \\
\hline 08-02-2008 & $\begin{array}{c}0,5 \% \\
(-0,1 \%, 1,1 \%)\end{array}$ & $\begin{array}{c}0,9 \% \\
(0,2 \%, 1,5 \%)\end{array}$ & $\begin{array}{c}1,5 \% \\
(0,5 \%, 2,4 \%)\end{array}$ & $\begin{array}{c}1,6 \% \\
(0,7 \%, 2,6 \%)\end{array}$ & $\begin{array}{c}1,5 \% \\
(0,7 \%, 2,3 \%)\end{array}$ & $\begin{array}{c}1,3 \% \\
(0,6 \%, 2 \%)\end{array}$ \\
\hline \multirow{2}{*}{$\begin{array}{c}\text { Choque } \\
\text { (dd-mm-aa) }\end{array}$} & \multicolumn{6}{|c|}{ VIRF para la variable RD } \\
\hline & Semana 1 & Semana 4 & Semana 13 & Semana 26 & Semana 39 & Semana 52 \\
\hline 09-08-2002 & $\begin{array}{c}9,6 \% \\
(-4 \%, 23,1 \%)\end{array}$ & $\begin{array}{c}10,9 \% \\
(-2,9 \%, 24,7 \%)\end{array}$ & $\begin{array}{c}11,2 \% \\
(-6,5 \%, 28,8 \%)\end{array}$ & $\begin{array}{c}9 \% \\
(-8,5 \%, 26,5 \%)\end{array}$ & $\begin{array}{c}6,8 \% \\
(-9,5 \%, 23,1 \%)\end{array}$ & $\begin{array}{c}5,1 \% \\
(-9,4 \%, 19,7 \%)\end{array}$ \\
\hline $26-12-2003$ & $\begin{array}{c}13,1 \% \\
(-5,6 \%, 31,8 \%)\end{array}$ & $\begin{array}{c}14,9 \% \\
(-5 \%, 34,8 \%)\end{array}$ & $\begin{array}{c}15,3 \% \\
(-8,2 \%, 38,8 \%)\end{array}$ & $\begin{array}{c}12,3 \% \\
(-10,7 \%, 35,4 \%)\end{array}$ & $\begin{array}{c}9,3 \% \\
(-12,2 \%, 30,8 \%)\end{array}$ & $\begin{array}{c}7 \% \\
(-12,3 \%, 26,3 \%)\end{array}$ \\
\hline $06-05-2005$ & $\begin{array}{c}13,9 \% \\
(-6,5 \%, 34,4 \%)\end{array}$ & $\begin{array}{c}15,9 \% \\
(-5 \%, 36,8 \%)\end{array}$ & $\begin{array}{c}16,3 \% \\
(-8,7 \%, 41,3 \%)\end{array}$ & $\begin{array}{c}13,1 \% \\
(-10,3 \%, 36,6 \%)\end{array}$ & $\begin{array}{c}9,9 \% \\
(-11,5 \%, 31,3 \%)\end{array}$ & $\begin{array}{c}7,5 \% \\
(-11,4 \%, 26,3 \%)\end{array}$ \\
\hline 22-09-2006 & $\begin{array}{c}7,6 \% \\
(-3,3 \%, 18,5 \%)\end{array}$ & $\begin{array}{c}8,7 \% \\
(-2,7 \%, 20 \%)\end{array}$ & $\begin{array}{c}8,9 \% \\
(-4,4 \%, 22,2 \%)\end{array}$ & $\begin{array}{c}7,2 \% \\
(-6 \%, 20,3 \%)\end{array}$ & $\begin{array}{c}5,4 \% \\
(-6,7 \%, 17,5 \%)\end{array}$ & $\begin{array}{c}4,1 \% \\
(-6,6 \%, 14,8 \%)\end{array}$ \\
\hline 08-02-2008 & $\begin{array}{c}8,5 \% \\
(-3,6 \%, 20,6 \%)\end{array}$ & $\begin{array}{c}9,7 \% \\
(-2,7 \%, 22,1 \%)\end{array}$ & $\begin{array}{c}10 \% \\
(-5 \%, 25 \%)\end{array}$ & $\begin{array}{c}8 \% \\
(-7,2 \%, 23,3 \%)\end{array}$ & $\begin{array}{c}6,1 \% \\
(-7,6 \%, 19,7 \%)\end{array}$ & $\begin{array}{c}4,6 \% \\
(-7,5 \%, 16,6 \%)\end{array}$ \\
\hline
\end{tabular}

\begin{tabular}{lcccccc}
\hline \multirow{2}{*}{$\begin{array}{c}\text { Choque } \\
\text { (dd-mm-aa) }\end{array}$} & VIRF para la variable RM \\
\cline { 2 - 7 } & Semana 1 & Semana 4 & Semana 13 & Semana 26 & Semana 39 & Semana 52 \\
\hline $09-08-2002$ & $128 \%$ & $105,9 \%$ & $56,3 \%$ & $17,6 \%$ & $5,6 \%$ & $8 \%$ \\
& $(96,3 \%, 159,8 \%)$ & $(81 \%, 130,7 \%)$ & $(28,9 \%, 83,7 \%)$ & $(-6 \%, 41,3 \%)$ & $(-11,5 \%, 22,6 \%)$ & $(-6,3 \%, 22,4 \%)$ \\
$26-12-2003$ & $169,3 \%$ & $140 \%$ & $74,4 \%$ & $23,3 \%$ & $7,4 \%$ & $10,6 \%$ \\
& $(130 \%, 208,7 \%)$ & $(107 \%, 173 \%)$ & $(38,4 \%, 110,5 \%)$ & $(-7,4 \%, 54,1 \%)$ & $(-15,6 \%, 30,4 \%)$ & $(-8,2 \%, 29,5 \%)$ \\
$06-05-2005$ & $134,8 \%$ & $111,5 \%$ & $59,3 \%$ & $18,6 \%$ & $5,9 \%$ & $8,5 \%$ \\
& $(102,6 \%, 167 \%)$ & $(84,2 \%, 138,7 \%)$ & $(29,4 \%, 89,1 \%)$ & $(-5,7 \%, 42,8 \%)$ & $(-11,3 \%, 23 \%)$ & $(-6,4 \%, 23,4 \%)$ \\
$22-09-2006$ & $134,9 \%$ & $111,5 \%$ & $59,3 \%$ & $18,6 \%$ & $5,9 \%$ & $8,5 \%$ \\
& $(102,1 \%, 167,6 \%)$ & $(84,5 \%, 138,5 \%)$ & $(31,4 \%, 87,1 \%)$ & $(-6,2 \%, 43,3 \%)$ & $(-13 \%, 24,7 \%)$ & $(-6,7 \%, 23,6 \%)$ \\
$08-02-2008$ & $153,9 \%$ & $127,2 \%$ & $67,6 \%$ & $21,2 \%$ & $6,7 \%$ & $9,7 \%$ \\
& $(119,6 \%, 188,1 \%)$ & $(99 \%, 155,4 \%)$ & $(34,2 \%, 101,1 \%)$ & $(-8,4 \%, 50,8 \%)$ & $(-14,8 \%, 28,2 \%)$ & $(-7,7 \%, 27,1 \%)$ \\
\hline
\end{tabular}

Nota: Función VIRF que muestra la respuesta en la desviación estándar de cada una de las series ante un choque de 25 p.b. en la tasa interbancaria (RM). Intervalos de confianza de $90 \%$ obtenidos a través de técnicas bootstrapping con 1.000 replicaciones. 
política monetaria resulta en un incremento positivo y significativo sobre la volatilidad de la tasa interbancaria, superior al $100 \%$ de la volatilidad observada antes del choque y su efecto ha desaparecido completamente alrededor de 20 semanas después. Sin embargo, la transmisión del choque hacia las demás tasas de interés es relativamente pequeña. En efecto, mientras que la respuesta de la volatilidad de la tasa de créditos ante un choque de política resulta en un incremento menor a $1 \%$ de la volatilidad observada antes del choque, la respuesta para la volatilidad de la tasa de depósitos resulta no significativa. Este resultado es de gran importancia ya que, en primer lugar, indica que la respuesta de la volatilidad del mercado ante un choque de política monetaria es relativamente pequeño, lo que evidencia que la credibilidad del banco central es alta y, además, resulta consistente con la magnitud de la transmisión de la tasa de intervención sobre la media de las otras tasas (Gráfico 2). Como se mencionó, una menor volatilidad en la tasa de interés incrementa la efectividad de la transmisión de choques de política.

Con el fin de verificar si el resultado anterior se mantiene a lo largo de la muestra, se estimaron las funciones VIRF y sus intervalos de confianza cada 73 semanas (aproximadamente una quinta parte de la muestra), resultados que se presentan en el Cuadro $1^{15}$. En general, aunque las magnitudes varían, las respuestas de la volatilidad de la tasa interbancaria y de las tasas de los créditos y los depósitos mantienen un comportamiento similar. En efecto, ante un choque monetario, la respuesta de la volatilidad de la tasa interbancaria se duplica en las primeras semanas después del choque, pero se disipa alrededor de 5 meses después. Por su parte, la respuesta de la volatilidad de la tasa de créditos exhibe un aumento significativo inferior a $2 \%$, mientras que el efecto en la varianza condicional de la tasa de depósitos no es significativo en ninguno de los períodos evaluados.

De esta manera, los análisis de impulso respuesta sugieren que la dinámica que exhibe el esquema de transmisión de política monetaria a través de las tasas de interés opera de manera apropiada. Por un lado, el efecto de un choque de política monetaria sobre la tasa interbancaria tiene el signo y la magnitud esperada por el banco central y su transmisión a las demás tasas de interés se consolida relativamente rápido, alrededor de tres meses después. Por otro lado, si bien un choque monetario afecta la volatilidad de la tasa de interés de los créditos de los bancos comerciales, su magnitud no es considerable, mientras que el efecto de un choque monetario sobre la volatilidad de la tasa de interés de los depósitos no es significativo.

\footnotetext{
${ }^{15}$ Vale la pena destacar que las conclusiones se mantienen cuando se realizan ejercicios similares a los presentados en el Cuadro 1 para fechas arbitrarias, en particular, fechas relacionadas con movimientos específicos de política monetaria.
} 


\section{CONCLUSIONES}

Este documento verifica la existencia de al menos un canal de transmisión de política monetaria en Colombia durante el período 2001-2008, tiempo en el cual la política monetaria se ha manejado bajo el esquema de metas de inflación.

En particular, este documento utiliza un modelo teórico simple en el cual es posible identificar las principales relaciones entre las tasas de interés de política monetaria, la tasa interbancaria y las tasas de depósitos y créditos, y posteriormente se incluyen consideraciones particulares que deben ser tenidas en cuenta bajo un esquema de metas de inflación. En particular, las decisiones de política monetaria en este esquema no solamente deben procurar mantener en un nivel apropiado su instrumento de política, generalmente la tasa de interés, sino que además se preocupa por mantener estabilidad en la economía, reduciendo la volatilidad de las tasas de interés.

En este orden de ideas, la dinámica de la transmisión de un choque de política monetaria sobre las tasas de interés es aproximada a través de modelos VECX-MGARCH, los cuales permiten no sólo evaluar el impacto de un choque de política monetaria en el nivel de las tasas de interés, sino que, además, incorpora la posibilidad de evaluar choques de política sobre la volatilidad de las tasas de interés.

En general, los resultados derivados del modelo econométrico son satisfactorios. En primer lugar, los resultados de las funciones de impulso respuesta indican que la transmisión de un choque en la tasa de interés de política es completa sobre las tasas de interés del mercado, entendiendo esto como una relación uno a uno entre la tasa subasta de expansión con la tasa de interés interbancario y las tasas de interés de los bancos comerciales. En efecto, un incremento de cien puntos básicos sobre la tasa subasta de expansión (el instrumento de política monetaria) se ve reflejado en un aumento de la misma magnitud sobre la tasa interbancaria (tasa objetivo de política) dos semanas después. Al igual que con la tasa interbancaria, un aumento en la tasa subasta de expansión tiene una relación uno a uno con las tasas de créditos y depósitos de los bancos comerciales, aunque en este caso dicha magnitud se observa cerca de un año después.

En segundo lugar, los análisis de impulso respuesta sobre la varianza condicional de las series, la respuesta de la volatilidad de las tasas de interés ante un choque de política monetaria sugieren que, si bien el banco central afecta la tasa de más corto plazo (la tasa interbancaria), su efecto sobre la tasa de créditos es relativamente pequeño y, en el caso de la tasa de depósitos, no significativo. Esto tiene importantes implicaciones para la evaluación de la efectividad de la política monetaria, ya que los resultados sugieren que la credibilidad y efectividad de las medidas del banco central son altas, en razón a que, por un lado, la transmisión del choque de política es completa y, por otro, su efecto sobre la volatilidad es reducido. 


\section{REFERENCIAS}

Amato, J. D. y T. Laubach (1999), "The value of interest rate smoothing: how the private sector helps the Federal Reserve". Economic Review, Federal Reserve Bank of Kansas City, N Q III, 47-64.

Bauwens, L., S. Laurent, S. y J.V.K. Rombouts (2006), "Multivariate GARCH models: a survey". Journal of Applied Econometrics, 21 (1): 79-109.

Bernanke, B. y A. Blinder (1992), "The Federal Funds Rate and the Channels of Monetary Transmission". The American Economic Review, 82 (4): 901-921.

Betancourt, R., N. Rodríguez, y H. Vargas (2008), "Interest rate pass-through in Colombia: a micro-banking perspective". Cuadernos de Economía (Latin American Journal of Economics) 45 (131): 29-58.

Bléjer, M. y A. Leone (2000), "Introduction and Overview", en: Bléjer, M., A. Ize, A.M. Leone, y S. Werlang (eds). Inflation Targeting in Practice: Strategic and Operational Issues and Application to Emerging Market Economies, 1-7. International Monetary Fund.

Blinder, A. S. (1999), "Central Bank Credibility: Why Do We Care? How Do We Build It?". NBER Working Paper No W7161.

Bollerslev, T. (1986), “Generalized autoregressive conditional Heterocedasticity”. Journal of Econometrics, 31: 307-327.

Bollerslev, T. (1990), "Modelling the Coherence in Short-Run Nominal Exchange Rates: A Multivariate Generalized ARCH Model". Review of Economics and Statistics, 72, 498-505.

Bollerslev, T., R.F. Engle y J. M. Wooldridge (1988), "A Capital Asset Pricing Model with Time-varying Covariances". Journal of Political Economy, 96 (1): $116-131$.

Boswijk, H. P. y J. A. Doornik (2005), "Distribution Approximations for Cointegration Tests with Stationary Exogenous Regressors". Journal of Applied Econometrics, 20 (6): 797-810.

Bredin, D., T. Fitzpatrick y G. O'Reilly (2002), "Retail Interest Rate Pass-Through: The Irish Experience". The Economic and Social Review, 33 (2): 223-246.

Dale, S. y A. G. Haldane (1995), "Interest rates and the channels of monetary transmission: Some sectoral estimates". European Economic Review, Elsevier, 39 (9): 1611-1626.

Engle, R. F. y K. F. Kroner (1995), "Multivariate Simultaneous Generalized ARCH". Econometric Theory, 11: 122-150.

Franses, P y D. van Dijk (2000), "Nonlinear Time Series Models in Empirical Finance". Cambridge.

Freixas, X. y C. Rochet (1997), “Microeconomics of Banking”. MIT Press.

Gómez, J., J.D. Uribe, y H. Vargas (2002), "The Implementation of Inflation Targeting in Colombia". Borradores de Economía Nº 202. Banco de la República.

Hafner, C. M. y H. Herwartz (2006), "Volatility impulse responses for multivariate GARCH models: An exchange rate illustration". Journal of International Money and Finance, 25 (5): 719-740.

Hannan, E. J. y B. G. Quinn (1979), “The Determination of the Order of an autoregression". Journal of the Royal Statistical Society. Series B (Methodological), 41 (2): 190-195

Huertas, C., M. Jalil, S. Olarte y J. Romero (2005), "Algunas Consideraciones sobre el Canal del Crédito y la Transmisión de Tasas de Interés en Colombia". Borradores de Economía No 351. Banco de la República. 
Hülsewig, O., E. Mayer y T. Wollmershauser (2006), "Bank loan supply and monetary policy transmission in Germany: An assessment based on matching impulse responses". Journal of Banking and Finance, Elsevier, 30 (10): 2893-2910.

Johansen, S. (1988), "Statistical analysis of cointegration vectors". Journal of Economic Dynamics and Control, 12: 231-254.

Johansen, S. (1991), "Estimation and Hypothesis Testing of Cointegration Vectors in Gaussian Vector Autoregressive Models". Econometrica, 59 (6): 1551-1580.

Kok Sorensen, C, y T. Werner (2006), "Bank interest rate pass-through in the euro area: a cross country comparison". Working Paper Series 580, European Central Bank.

Longworth, D. (2000), “The Canadian Monetary Transmission Mechanism”, en Blejer, M., A. Ize, A.Leone y S. Werlang. (eds). Inflation Targeting in Practice: Strategic and Operational Issues and Application to Emerging Market Economies, 37-43. International Monetary Fund.

Lütkepohl, H. (2004), “Applied Time Series Econometrics”, Cambridge University Press.

Lütkepohl, H. (2005), “New Introduction to Multiple Time Series Analysis”. Springer.

Mishkin, F. (1996), "The Channels of Monetary Transmission: Lessons for Monetary Policy". NBER Working Paper Series 5464.

Mojon, B. (2000), "Financial structure and the interest rate channel of ECB monetary policy". Working Paper 40, European Central Bank.

Schwarz, G. (1978), "Estimating the dimension of a model". The Annals of Statistics. Vol. 6, No 2, 461-464.

Tuysuz, S. (2007), "The effects of a greater central bank credibility on interest rates level and volatility response to news in the U.K.", University Library of Munich, Germany, MPRA Paper 5263.

Weth M. A. (2002), "The pass-through from market interest rates to bank lending rates in Germany". Discussion Paper 11/02, Deutsche Bundesbank. 


\section{Anexo A \\ TASAS DE INTERÉS UTILIZADAS EN EL DOCUMENTO}

Para evaluar la transmisión de un choque de política monetaria sobre las demás tasas de interés, se utilizaron cuatro tasas, la tasa subasta de expansión $\left(r^{S}\right)$, la tasa interbancaria $\left(r^{M}\right)$, la tasa de certificados de depósitos a término fijo de 90 días $\left(r^{D}\right)$ y la tasa de colocación sin tesorería $\left(r^{L}\right)$ durante el período comprendido entre enero de 2001 y febrero de 2008, todas expresadas en términos nominales, en frecuencia semanal. A continuación, se describen las principales generalidades y metodologías de cálculo para cada una de las tasas anteriores ${ }^{16}$.

\section{Tasas de intervención del Banco de la República}

- Tasas subasta de expansión (contracción): Son las tasas a las cuales el banco central aumenta (o contrae) liquidez del mercado a través de subastas en las que se ha fijado previamente el monto.

- Tasa lombardas de expansión (contracción): Es la máxima (mínima) tasa a la cual el banco central expande (contrae) la liquidez del sistema financiero a través de ventanillas en las que se retiran (depositan) montos ilimitados.

Cabe anotar que de estas cuatro tasas, la única tasa que se ha mantenido activa durante todo el período ha sido la tasa subasta de expansión. En efecto, las tasas subasta de contracción, lombarda de expansión y lombarda de contracción han sido cerradas discrecionalmente por el banco central en algunos períodos de la muestra.

Tasa interbancaria (TIB): Es la tasa (para un día hábil) a la que negocian los intermediarios financieros entre sí, y no requiere garantía. Se calcula como el promedio ponderado por monto de las tasas negociadas en el día.

Tasa de certificados de depósitos a término fijo a 90 días (DTF): Es el promedio de las tasas de interés efectivas de los certificados de depósito a término fijo (CDT) a 90 días, ponderado por monto transado, emitidos por los bancos, corporaciones financieras y compañías de financiamiento comercial. Es calculada por el BR como resultado de todas las operaciones reportadas por los intermediarios financieros a lo largo de una semana que va de viernes a jueves.

16 Adaptado de http://www.banrep.gov.co/series-estadisticas/see_glosa.htm\#15. 
Tasa de colocación ( $\sin$ tesorería): La tasa de colocación sin tesorería se calcula como el promedio ponderado por monto transado de las tasas activas de los establecimientos de crédito (bancos, corporaciones financieras, compañías de financiamiento comercial, organismos cooperativos y cooperativas financieras). De acuerdo con la legislación colombiana actual, los créditos de estos establecimientos se agrupan en cuatro diferentes categorías:

- $\quad$ Tasa de crédito preferencial: Es la tasa cobrada a aquellos clientes preferenciales o corporativos que tienen poder de negociación, donde la categoría preferencial implica una menor exposición al riesgo de crédito y, por ende, una tasa relativamente menor a las demás. Incluye las tasas implementadas por todos los establecimientos de crédito.

- Tasa de crédito de tesorería: Es la tasa cobrada a los créditos otorgados a clientes preferenciales o corporativos con un plazo inferior a 30 días, cuyo objetivo primordial es el de solucionar eventuales problemas de liquidez.

- Tasa de crédito de consumo: Es la tasa cobrada a los créditos otorgados a particulares por parte de los establecimientos de crédito, cuyo fin es la adquisición de bienes de consumo o el pago de servicios no comerciales. No se incluyen préstamos con tarjeta de crédito. Se incluyen todos los montos y plazos reportados.

- Tasa de crédito ordinario: La tasa de crédito ordinario está asociada con los créditos que no pueden ser clasificados como de Tesorería o Preferencial. Incluye todos los montos y plazos posibles.

La tasa de colocación sin tesorería es el promedio, ponderado por monto, de las tasas de crédito de consumo, preferencial y ordinario de los días hábiles correspondientes a los anteriores 30 días calendario reportado por los establecimientos de crédito, excluyendo los créditos con una duración inferior a 90 días. Adicionalmente, como su nombre lo indica, esta tasa excluye de su cálculo todos los préstamos asociados con créditos de tesorería. 


\section{ANEXo B}

\section{PRUEBAS DE ESPECIFICACIÓN}

CUADRO B-1

PRUEBAS DE RAIIZ UNITARIA

\begin{tabular}{clccc}
\hline \multicolumn{1}{c}{ Prueba } & Hipótesis Nula & Estadística & $\begin{array}{c}\text { Valor Crítico } \\
\text { al 5\% }\end{array}$ \\
\hline$r^{L}$ & $\begin{array}{l}\text { Elliott-Rothenberg-Stock } \\
\text { DF con constante }\end{array}$ & No estacionariedad & $-0,040$ & $-1,942$ \\
\hline$r^{L}$ & KPSS con constante & Estacionariedad & 1,808 & 0,463 \\
\hline$r^{D}$ & $\begin{array}{l}\text { Elliott-Rothenberg-Stock } \\
\text { DF con constante }\end{array}$ & No estacionariedad & $-0,006$ & $-1,942$ \\
\hline$r^{D}$ & KPSS con constante & Estacionariedad & 1,692 & 0,463 \\
\hline \multirow{2}{*}{$r^{M}$} & $\begin{array}{l}\text { Elliott-Rothenberg-Stock } \\
\text { DF con constante }\end{array}$ & No estacionariedad & $-0,182$ & $-1,942$ \\
\hline$r^{M}$ & KPSS con constante & Estacionariedad & 0,509 & 0,463 \\
\hline
\end{tabular}

CUADRO B-2

PRUEBAS DE COINTEGRACIÓN DE JOHANSEN

\begin{tabular}{cccc}
\hline $\begin{array}{c}\text { Ho: Rango } \\
\text { de } \Pi\end{array}$ & Traza & $\begin{array}{c}\text { Estadística Q } \\
\text { (Boswijk y Doornik) }\end{array}$ & $\begin{array}{c}\text { Valores críticos al } \\
10 \% \text { (est. Q) }\end{array}$ \\
\hline 0 & 31,16 & 16,067 & 12,861 \\
1 & 14,38 & 6,987 & 6,049 \\
2 & 3,17 & 1,714 & 2,289 \\
\hline
\end{tabular}

CUADRO B-3

VECTORES DE COINTEGRACIÓN ESTIMADOS

\begin{tabular}{ccc}
\hline Variables & Primer vector de cointegración & Segundo vector de cointegración \\
\hline$r^{L}$ & 1 & 0 \\
$r^{D}$ & 0 & 1 \\
$r^{M}$ & $-1,592$ & $-1,540$ \\
& $(0,285)$ & $(0,210)$ \\
\hline
\end{tabular}

* Errores estándar entre paréntesis. 


\section{CUADRO B-4 \\ PRUEBAS DE ESPECIFICACIÓN SOBRE LOS RESIDUALES \\ DEL MODELO VECX $(10,4)$}

\begin{tabular}{|c|c|c|c|c|}
\hline \multirow[b]{2}{*}{ Prueba } & \multicolumn{4}{|c|}{ Pruebas Univariadas } \\
\hline & Hipótesis Nula & Rezagos & Estadística & Valor $\mathrm{p}$ \\
\hline $\begin{array}{l}\text { Prueba de Engle sobre } \\
\text { ecuación } r^{L}\end{array}$ & $\begin{array}{l}\text { No hay efecto } \\
\text { GARCH }\end{array}$ & 15 & 22,79 & 0,09 \\
\hline $\begin{array}{l}\text { Prueba de Engle sobre } \\
\text { ecuación } r^{D}\end{array}$ & $\begin{array}{c}\text { No hay efecto } \\
\text { GARCH }\end{array}$ & 15 & 29,44 & 0,01 \\
\hline \multirow[t]{3}{*}{$\begin{array}{l}\text { Prueba de Engle sobre } \\
\text { ecuación } r^{M}\end{array}$} & $\begin{array}{c}\text { No hay efecto } \\
\text { GARCH }\end{array}$ & 15 & 68,86 & $<0,001$ \\
\hline & \multicolumn{4}{|c|}{ Pruebas Multivariadas } \\
\hline & Hipótesis Nula & Rezagos & Estadística & Valor $\mathrm{p}$ \\
\hline $\begin{array}{l}\text { Portmanteau tipo } \\
\text { Ljung-Box sobre los } \\
\text { residuales }\end{array}$ & $\begin{array}{c}\text { No existe } \\
\text { autocorrelación }\end{array}$ & 90 & 656,69 & 0,99 \\
\hline $\begin{array}{l}\text { Portmanteau tipo } \\
\text { Ljung-Box sobre el } \\
\text { cuadrado de los } \\
\text { residuales }\end{array}$ & $\begin{array}{l}\text { No hay efecto } \\
\text { MGARCH }\end{array}$ & 15 & 529,60 & $<0,001$ \\
\hline
\end{tabular}

\section{CUADRO B-5. PRUEBAS DE ESPECIFICACIÓN \\ MULTIVARIADAS SOBRE LOS RESIDUALES ESTANDARIZADOS DEL MODELO VECX $(10,4)-M G A R C H(1,1)$}

\begin{tabular}{lcccc}
\hline \multicolumn{1}{c}{ Prueba } & Hipótesis Nula & Rezagos & Estadística & Valor p \\
\hline $\begin{array}{l}\text { Portmanteau tipo } \\
\begin{array}{l}\text { Ljung-Box sobre } \\
\text { los residuales }\end{array}\end{array}$ & $\begin{array}{c}\text { No existe } \\
\text { autocorrelación }\end{array}$ & 90 & 765,15 & 0,87 \\
\hline $\begin{array}{l}\text { Portmanteau tipo } \\
\text { Ljung-Box sobre el } \\
\text { cuadrado de los residuales }\end{array}$ & $\begin{array}{c}\text { No hay efecto } \\
\text { MGARCH }\end{array}$ & 15 & 149,11 & 0,19 \\
\hline Shapiro & Normalidad & - & 0,975 & $<0,001$ \\
\hline
\end{tabular}

\section{CUADRO B-6 \\ MÓDULOS DE LAS RAÍCES DE LOS POLINOMIOS ASOCIADOS AL MODELO $\operatorname{VECX}(10,4)-M G A R C H(1,1)^{*}$}

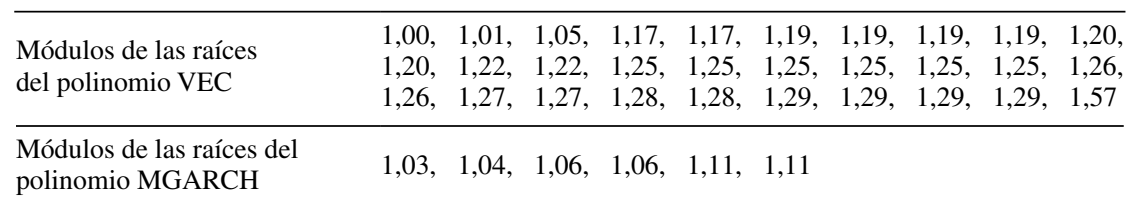

* Módulos menores que uno implican no estacionariedad. 


\section{Anexo C \\ MODELOS GARCH MULTIVARIADOS}

La extensión multivariada de los modelos GARCH corresponde a una generalización de los modelos univariados desarrollados por Bollerslev (1986). En el caso multivariado, la media condicional del proceso $y_{t}$ de dimensión $N \times 1$ puede ser representada por un modelo $\operatorname{VARX}(l, s)$ :

$$
y_{t}=\mu+C_{1} y_{t-1}+\ldots+C_{l} y_{t-l}+D_{0} x_{t}+\ldots+D_{s} x_{t-s}+\varepsilon_{t}
$$

Donde $x_{t}$ corresponde a un vector de variables exógenas de dimensión $M$ y $C_{i}$ y $D_{i}$ representan matrices de coeficientes de dimensiones $N \times N$ y $N \times M$, respectivamente.

Usualmente, se asume que los errores del modelo (C1) tienen una distribución condicional normal multivariada con valor esperado cero (vectorial) y matriz de covarianzas $H_{t}$. Si $\psi_{t}$ denota el conjunto de información disponible hasta el $t$ :

$$
\varepsilon_{t} \mid \psi_{t-1} \sim N_{N}\left(0, H_{t}\right)
$$

Este modelo es denominado VARX - MGARCH. Al igual que en el modelo $\operatorname{GARCH}(p, q)$ del caso univariado, la matriz de covarianzas $H_{t}$ depende de las observaciones pasadas de $\varepsilon_{t-i}, i=1, \ldots, q$ y de las matrices de covarianzas $H_{t-i}$, $i=1, \ldots, p$. Considerando que vech $(\cdot)$ denota el operador que transforma la parte triangular inferior (incluyendo la diagonal) de una matriz simétrica $N \times N$ en un vector de dimensión $N^{*}=(N(N+1) / 2)$, un modelo $\operatorname{GARCH}(p, q)$ mutivariado, $\operatorname{MGARCH}(p, q)$, puede ser representado como:

(C3) $\operatorname{vech}\left(H_{t}\right)=c+\sum_{i=1}^{q} A_{i} \operatorname{vech}\left(\varepsilon_{t-i} \varepsilon_{t-i}^{\prime}\right)+\sum_{i=1}^{p} B_{i} \operatorname{vech}\left(H_{t-i}\right)$

La expresión (C3) es denominada representación vec del modelo $M G A R C H^{17}$, $A_{i}$ y $B_{i}$ corresponden a matrices de parámetros de dimensión $N^{*} \times N^{*}$ y $c$ es un vector $N^{*} \times 1$.

Una condición suficiente para la estacionariedad en sentido débil del proceso $\left\{\varepsilon_{t}\right\}$ se tiene si todas las raíces del determinante de la ecuación matricial (4) están por fuera del círculo unitario:

$$
\operatorname{det}\left[I-\sum_{i=1}^{\max (p, q)}\left(A_{i}+B_{i}\right) z^{i}\right]=0
$$

${ }^{17}$ Bollerslev et al. (1988). 
Con $A_{q+1}=\ldots=A_{p}=0$ si $p>q$, y $B_{p+1}=\ldots=B_{q}=0$ si $q>p$.

Bajo el supuesto de estacionariedad débil, la matriz de covarianzas no condicionada de $\varepsilon_{t}$ es la siguiente:

$$
\operatorname{vech}(H)=\left(I-\sum_{i=1}^{\max (p, q)}\left(A_{i}+B_{i}\right)\right)^{-1} c
$$

Un problema del modelo $\operatorname{MGARCH}(p, q)$ vec es su alto número de parámetros, $\frac{1}{4}(p+q) N^{2}(N+1)^{2}+\frac{1}{2} N(N+1)$; por lo cual, se han propuesto varias alternativas; entre los cuales se encuentran modelos diagonales y modelos basados en $G A R C H$ univariados ${ }^{18}$.

Bollerslev et al. (1988) propusieron los modelos vec diagonales, o dvec, en los cuales las matrices $A_{i}$ y $B_{i}$ son diagonales. Sin embargo, bajo esta representación no se puede garantizar que la matriz de covarianzas $H_{t}$ sea definida positiva.

Otra manera de formular los modelos $M G A R C H$ es a través de modelos GARCH univariados. Uno de estos casos corresponde al modelo de correlación constante, $C C C$, sugerido por Bollerslev (1990). Este modelo parte del hecho que $H_{t}=\Delta_{t} R_{t} \Delta_{t}$, donde $R_{i}$ corresponde a la matriz de correlación condicional y $\Delta_{t}$ es a la matriz diagonal de desviaciones estándar condicionales.

El modelo $C C C$ supone que la matriz de correlaciones condicionales es constante en el tiempo y, por lo tanto, $H_{t}=\Delta_{t} R \Delta_{t}$. En esta representación Bollerslev supone que las varianzas condicionales son determinadas de acuerdo a modelos GARCH univariados, mientras que las covarianzas condicionales son determinadas por la relación $H_{t}=\Delta_{t} R \Delta_{t}$.

Engle y Kroner (1995) plantearon la siguiente especificación:

$$
H_{t}=C_{0}^{\prime} C_{0}+\sum_{k=1}^{K} \sum_{i=1}^{q} F_{k i}^{\prime} \varepsilon_{t-i} \varepsilon_{t-i}^{\prime} F_{k i}+\sum_{k=1}^{K} \sum_{i=1}^{p} G_{k i}^{\prime} H_{t-i} G_{k i}
$$

donde $C_{0}$ es una matriz triangular inferior de orden $N \times N$ y $F_{k i}$ y $G_{k i}$ son matrices $N \times N$ de parámetros.

Este modelo es conocido como representación $B E K K(p, q, k)$. En su forma más sencilla, $\operatorname{BEKK}(p, q, 1)$, este modelo tiene menos parámetros que los modelos vec y presenta dos características positivas. Primero, las matrices $H_{t}$ son simétricas y definidas positivas si por lo menos alguna de las matrices $C_{0}$ ó $G_{k i}$ tiene rango completo. Segundo, a diferencia de otros modelos más restrictivos, la representación $B E K K$ permite una mayor dinámica. Por ejemplo, no asume que la matriz de

\footnotetext{
18 Sin embargo, existen otras representaciones de modelos MGARCH. Por ejemplo, generalizaciones de modelos de correlación constante, modelos factoriales, modelos con coeficientes aleatorios, entre otros. Un recuento de este tipo de modelos puede ser consultado en Franses y van Dijk (2000) y Bauwens et al. (2006).
} 
correlaciones condicional sea constante; adicionalmente, este modelo permite una dependencia directa entre la varianza condicional de una variable con respecto a los datos observados de las varianzas condicionales de otras variables del sistema.

El modelo $B E K K(p, q, k)$ puede ser representado en la notación vec utilizando las propiedades de los operadores vech y $v e c^{19}$. Para $p=q=k=1$ este modelo se representa como:

$$
\begin{aligned}
& \begin{aligned}
H_{t}=C_{0}^{\prime} C_{0}+F_{1}^{\prime} \varepsilon_{t-1} \varepsilon_{t-1}^{\prime} F_{1}+G_{1}^{\prime} H_{t-1} G_{1} \\
\operatorname{vech}\left(H_{t}\right)=\operatorname{vech}\left(C_{0}^{\prime} C_{0}\right)+L_{N} \operatorname{vec}\left(F_{1}^{\prime} \varepsilon_{t-1} \varepsilon_{t-1}^{\prime} F_{1}\right)+L_{N} \operatorname{vec}\left(G_{1}^{\prime} H_{t-1} G_{1}\right) \\
\operatorname{vech}\left(H_{t}\right)=\operatorname{vech}\left(C_{0}^{\prime} C_{0}\right)+L_{N}\left(F_{1} \otimes F_{1}\right)^{\prime} D_{N} \operatorname{vech}\left(\varepsilon_{t-1} \varepsilon_{t-1}^{\prime}\right)+ \\
+L_{N}\left(G_{1} \otimes G_{1}\right)^{\prime} D_{N} \operatorname{vech}\left(H_{t-1}\right)
\end{aligned}
\end{aligned}
$$

donde $D_{N}$ representa la matriz de duplicación tal que para una matriz simétrica $A$ de dimensiones $N \times N$, vec $(A)=D_{N}$ vech $(A)$ y $L_{N}$ es la matriz de eliminación tal que $\operatorname{vech}(A)=L_{N} \operatorname{vec}(A)$.

${ }^{19} \mathrm{El}$ operador vec transforma una matriz en un vector posicionando las columnas de la matriz una debajo de otra. 


\section{Anexo D}

\section{FUNCIÓN DE IMPULSO RESPUESTA EN VOLATILIDAD}

En los modelos de series de tiempo multivariados la función de impulso respuesta se utiliza para analizar el efecto de un choque a una serie del sistema. Este concepto está asociado al modelamiento del primer momento de las series y puede ser generalizado al segundo momento usando modelos GARCH multivariados. Es así como Hafner y Herwartz (2006) definen la función de impulso respuesta en la volatilidad, VIRF por sus siglas en inglés, para un modelo MGARCH como la diferencia entre el valor esperado condicional de $H_{t}$ con y sin choque ${ }^{20}$ :

$$
\operatorname{VIRF}_{t}(\delta)=E\left[\operatorname{vech}\left(H_{t}\right) \mid \varepsilon_{0}=\delta, \Psi_{-1}\right]-E\left[\operatorname{vech}\left(H_{t}\right) \mid \varepsilon_{0}=0, \Psi_{-1}\right]
$$

Por lo tanto, la VIRF indica el efecto que tiene un choque de magnitud $\delta$ sobre la varianza condicional, $t$ períodos después de haberse realizado. Para un modelo $\operatorname{MGARCH}(1,1)$ en representación vec se tiene el siguiente resultado:

(D2) $\quad \operatorname{VIRF}_{t}(\delta)=\left\{\begin{array}{c}A_{1} \text { vech }\left(\delta \delta^{\prime}\right) \text { para } t=1 \\ \left(A_{1}+B_{1}\right) V I R F_{t-1}(\delta) \text { para } t>1\end{array}\right.$

Utilizando la expresión (C7), la VIRF para un modelo $\operatorname{BEKK}(1,1,1)$ está dada por:

(D3) $\quad \operatorname{VIRF}_{t}(\delta)=\left\{\begin{array}{c}L_{N}\left(F_{1} \otimes F_{1}\right)^{\prime} D_{N} \text { vech }\left(\delta \delta^{\prime}\right) \text { para } t=1 \\ L_{N}\left(F_{1} \otimes F_{1}+G_{1} \otimes G_{1}\right)^{\prime} D_{N} V I R F_{t-1}(\delta) \text { para } t>1\end{array}\right.$

Hafner y Herwartz proponen dividir cada elemento de la VIRF por las volatilidades condicionales asociadas al de choque, $H_{0}$. Esto permite interpretar estos resultados como desviaciones porcentuales con respecto a los elementos de $H_{0}$.

Una diferencia importante entre la función de impulso respuesta para un modelo VAR o VARX y la VIRF estandarizada, es que esta última sí depende del en que se realiza el choque. Esto es debido a que la VIRF estandarizada depende de $H_{0}$.

${ }^{20}$ Hafner y Herwartz (2006) utilizan varias definiciones de VIRF. En este documento se utiliza la definición que los autores denominan como $C V P$, la cual es la más cercana a la definición de la función de impulso respuesta tradicional. Se usa la versión con choque fijo y con línea de comparación cero. 